Stephens, R.S., Roffman, R.A., \& Curtin, L. (2000). Comparison of extended versus brief treatments for marijuana use. Journal of Consulting and Clinical Psychology, 68, 898-908. American Psychological Association (ISSN: 0022-006X) Submitted: June 8, 1999 Revised: February 7, 2000 Accepted: February 11, 2000 DOI: 10.1037/0022-006X.68.5.898 'This article may not exactly replicate the final version published in the APA journal. It is not the copy of record.

\title{
Comparison of Extended Versus Brief Treatments for Marijuana Use.
}

\author{
Robert S. Stephens, Roger A. Roffman \& Lisa Curtin
}

\begin{abstract}
Adult marijuana users $(\mathrm{N}=291)$ seeking treatment were randomly assigned to an extended 14session Cognitive-behavioral group treatment (relapse prevention support group; RPSG), a brief 2 -session individual treatment using motivational interviewing (individualized assessment and intervention; IAI), or a 4-month delayed treatment control (DTC) condition. Results indicated that marijuana use, dependence symptoms, and negative consequences were reduced significantly in relation to pretreatment levels at 1-, 4-, 7-, 13-, and 16-month follow-ups. Participants in the RPSG and IAI treatments showed significantly and substantially greater improvement than DTC participants at the 4-month follow-up. There were no significant differences between RPSG and IAI outcomes at any follow-up. The relative efficacy of brief versus extended interventions for chronic marijuana-using adults is discussed.
\end{abstract}

Keywords: Cognitive Therapy | Individual Psychotherapy | Marijuana Usage | Motivational Interviewing | Treatment Duration | Brief Psychotherapy

Acknowledgement: This research was supported by National Institute on Drug Abuse Grant 2 R01 DA03586-04. We thank Bonnie Cleaveland, Deborah DeWolfe, and Jennifer Wertz for important contributions to the conduct of this study. 


\begin{abstract}
ARTICLE
In $1997,25 \%$ of the admissions to state-funded drug abuse clinics for illicit substance use were for marijuana abuse, a proportion comparable to that for cocaine (29\%) and heroin (29\%) abuse in these same settings. These rates represent a nearly two-fold increase in the demand for marijuana treatment since 1992 (Substance Abuse and Mental Health Services Association, Office of Applied Studies, 1999). Epidemiological studies indicate that the relative risk for a lifetime diagnosis of cannabis dependence is slightly more than $4 \%$ in the U.S. populationhighest for any illicit drug (Anthony \& Helzer, 1991; Anthony, Warner, \& Kessler, 1994). As many as $9 \%$ of those who have used marijuana at least once will qualify for the dependence diagnosis at some time in their life (Anthony et al., 1994). The relative risk of dependence may be as high as $20 \%-30 \%$ for those who have used marijuana more than a few times (Hall, Solowij, \& Lemon, 1994). There is evidence that users are particularly likely to seek treatment when interventions are tailored specifically to marijuana use problems (Stephens \& Roffman, 1993; Stephens, Roffman, \& Simpson, 1993). Despite the large number of marijuana users who may want or need help, there are still surprisingly few data on treatment efficacy.
\end{abstract}

The long-term health consequences of heavy marijuana use are only beginning to be understood, but recent reviews suggest several areas for concern (Hall et al., 1994; Stephens, 1999). There is considerable evidence of the detrimental effects of smoked marijuana on the respiratory system (Tashkin et al., 1990). Researchers have identified impairments in the attentional and executive functioning of heavy marijuana users that do not show up in more global estimates of intelligence (Pope \& Yurgelun-Todd, 1996; Solowij, Michie, \& Fox, 1991, 1995). Similar deficits may occur in the children of mothers who smoked marijuana during pregnancy (Day et al., 1994; Fried, 1995; Richardson, Day, \& Goldschmidt, 1995). Chronic, heavy marijuana users report impairments of memory, concentration, motivation, self-esteem, interpersonal relationships, health, employment, or finances related to their marijuana use (Haas \& Hendin, 1987; Rainone, Deren, Kleinman, \& Wish, 1987). Adults who sought treatment in a prior controlled treatment trial averaged over 10 years of near-daily use and over six serious attempts at quitting (Stephens et al., 1993). Their use persisted despite negative consequences, and most perceived themselves as unable to stop. Furthermore, these negative consequences were not explained by concurrent alcohol or other drug abuse (Stephens et al., 1993). 
In the only published controlled treatment trial (Stephens, Roffman, \& Simpson, 1994), a cognitive-behavioral group treatment that focused on relapse prevention (RP; Marlatt \& Gordon, 1985) was compared with a group intervention that focused on social support and discussion of issues related to quitting. Participants were daily marijuana users who voluntarily sought treatment after many years of chronic use. Outcome data collected for 12 months posttreatment failed to show any significant differences between the treatment conditions. Approximately $65 \%$ of the participants quit using by the end of treatment, and the frequency of use was significantly reduced from baseline at all follow-up assessments. Twelve months after treatment, approximately $17 \%$ were abstinent, illustrating that relapse rates were large and similar to those typically found for alcohol, tobacco, and other drugs of abuse.

The results of this study were encouraging regarding the appeal and potential efficacy of treatments for marijuana use and related problems, but the lack of differential outcomes limited conclusions regarding the causal role of treatment (Stephens et al., 1994). Although substantial reductions in marijuana use were observed in a chronic marijuana-using sample, it was possible that these outcomes were primarily a function of the motivated, self-referred sample rather than the treatments. The failure to find superior outcomes for participants who received the RP treatment also was counter to expectations and raised a concern that 3 months of treatment was inadequate for the acquisition of cognitive-behavioral coping skills needed to avoid relapse.

On the basis of these findings, the present study had three main purposes. First, we wanted to examine whether extending the RP group treatment by adding additional sessions, extending social support, and involving significant others (e.g., McCrady, 1989; O'Farrell, Choquette, Cutter, Brown, \& McCourt, 1993) would increase abstinent rates and improve overall outcomes. Second, we wanted to examine the effects of very brief treatment in reducing marijuana use. Recent reviews (e.g., Bien, Miller, \& Tonigan, 1993) and large sample effectiveness research (e.g., Fleming, Barry, Manwell, Johnson, \& London, 1997) concluded that treatments of one to three sessions are often as effective as longer, more intensive treatments in producing changes in alcohol use. Brief interventions have not been studied in the treatment of illicit substance abuse, and they offer the potential for cost-effective intervention. Third, we wanted to rule out the participants' initial motivation for change or reactivity to the assessment procedures as the explanations for posttreatment reductions in marijuana use. Thus, we included a delayed treatment or wait-list condition to assess change in the absence of intervention.

We hypothesized that the extended group treatment would result in higher rates of abstinence and greater reductions in marijuana use, dependence symptoms, and associated problems when compared with the delayed treatment and brief intervention conditions. The increased number of 
treatment sessions was expected to allow for greater coping skill acquisition and extended social support. The involvement of significant others also was expected to enhance motivation for change and help protect against relapse during the follow-up period. On the basis of research with alcohol abusers, we also hypothesized that the brief intervention would produce superior outcomes on these same variables when compared with the delayed treatment condition.

\section{Method}

\section{Participants}

Of the 601 potential participants who were screened for participation over a 22-month period, 183 were ineligible to participate because they had used marijuana less than 50 times in the past 90 days $(n=24)$, reported alcohol or other drug abuse during the past 90 days $(n=149), 1$ reported severe psychological distress $(n=8$; e.g., suicidal intentions or psychotic thought processes), or were involved in other formal treatment for marijuana abuse $(n=2)$. These eligibility criteria were similar to those used in prior research (Stephens et al., 1994) and were chosen to yield a sample of relatively pure marijuana users appropriate for outpatient interventions. Of the 418 eligible participants, 127 failed to complete pretreatment assessment and research protocols. The 291 participants randomly assigned to treatment conditions averaged 34.0 years of age $(\mathrm{SD}=6.85)$ and had completed 14 years of education $(\mathrm{SD}=2.80)$. They were largely male (77\%), Caucasian (95\%), employed full-time (76\%), and single (55\%).

\section{Design}

Participants were randomly assigned to one of three treatment conditions: relapse prevention support groups (RPSGs; $\mathrm{n}=117$ ), individualized assessment and advice (IAI; $\mathrm{n}=88$ ), and delayed treatment control (DTC; $n=86$ ). 2 Follow-up assessments were scheduled in relation to the start of treatment (i.e., 1, 4, 7, 13, and 16 months after random assignment; see Table 1) in order to control for the effects of history when comparing treatments of different lengths. Follow-up assessment points were chosen such that the IAI and RPSG treatment participants were assessed at the end of treatment, 3 months posttreatment, and 12 months posttreatment, thus providing common reference points in relation to the end of treatment. The DTC participants were reassessed only at the 4-month follow-up to provide a no treatment comparison corresponding to the end of treatment for RPSG participants and to the 3-month posttreatment point for IAI participants. DTC participants were not assessed at the 1-month follow-up in order to minimize reactivity effects. DTC participants were provided treatment following the initial waiting period, but their posttreatment outcomes were not assessed. 
Table 1

Study Design and Follow-Up Rates

\begin{tabular}{|c|c|c|c|c|c|c|c|c|c|c|c|c|c|c|c|}
\hline \multirow[b]{3}{*}{$\begin{array}{l}\text { Treatment } \\
\text { condition }\end{array}$} & \multicolumn{15}{|c|}{ Follow-up assessments } \\
\hline & \multicolumn{3}{|c|}{1 month } & \multicolumn{3}{|c|}{4 months } & \multicolumn{3}{|c|}{7 months } & \multicolumn{3}{|c|}{13 months } & \multicolumn{3}{|c|}{16 months } \\
\hline & $\begin{array}{c}\text { Reference } \\
\text { point }\end{array}$ & $n$ & $\%$ & $\begin{array}{c}\text { Reference } \\
\text { point }\end{array}$ & $n$ & $\%$ & $\begin{array}{c}\text { Reference } \\
\text { point }\end{array}$ & $n$ & $\%$ & $\begin{array}{c}\text { Reference } \\
\text { point }\end{array}$ & $n$ & $\%$ & $\begin{array}{c}\text { Reference } \\
\text { point }\end{array}$ & $n$ & $\%$ \\
\hline RPSG $(N=117)$ & Quit date & 99 & 85 & End treatment & 95 & 81 & 3 months post & 95 & 81 & 9 months post & 101 & 86 & 12 months post & 103 & 88 \\
\hline IAI $(N=88)$ & End treatment & 81 & 92 & 3 months post & 75 & 85 & 6 months post & 72 & 82 & 12 months post & 78 & 89 & 15 months post & 80 & 91 \\
\hline DTC $(N=86)$ & & & & End of delay & 79 & 92 & & & & & & & & & \\
\hline
\end{tabular}

Note. The 1-, 4-, 7-, 13-, and 16-month follow-up assessments are labeled in relation to time elapsed since the start of treatment or random assignment to DTC. Table entries indicate treatment-specific reference points. Post $=$ months since treatment ended. $N \mathrm{~s}$ and percentages represent the numbers and proportions of the randomized samples completing each assessment. RPSG $=$ relapse prevention support group; $\mathrm{IAI}=$ individualized assessment and advice; DTC $=$ delayed treatment control.

The inclusion of a delayed treatment condition raised ethical concerns regarding the withholding of treatment. Several circumstances seemed to justify the inclusion of this control condition. First, neither the RPSG nor the IAI treatments had been tested in controlled trials and were not known to be effective, either in the absolute sense or relative to other treatment available within the community. Second, the sample to be treated consisted of adults who had been smoking marijuana almost daily for 15 or more years without imminent life-threatening consequences. The increase in risk associated with waiting 4 months for treatment appeared negligible. Third, informed consent, the offer of referrals to other treatment, and the absence of any prohibition against seeking treatment during the waiting period ensured that potential participants could obtain treatment without waiting.

\section{Pretreatment Procedures}

Media announcements, news stories, and paid advertisements in local newspapers and on radio stations in the greater Seattle, Washington, area promoted the Marijuana Treatment Project for adult marijuana users who wanted help quitting. Respondents were screened on the phone for age and then scheduled for small group or individual orientation sessions that lasted approximately $2 \mathrm{hr}$. During the orientation session, the participant reviewed an informed consent form that included the following: a brief description of the treatment conditions, the nature of random assignment, the requirement of a $\$ 60$ deposit to be refunded on completion of the follow-ups (i.e., \$10 refunded for completion of each of the first four follow-ups and $\$ 20$ for the final follow-up), and the need to identify a collateral informant who would be contacted independently at all follow-up assessments. The confidentiality of all records was emphasized, and participants were informed that the project was covered by a Certificate of Confidentiality from the U.S. Department of Health and Human Services that protected the investigators from being compelled to identify research participants. 
Participants were told that the RPSG and IAI treatments were designed to produce abstinence from marijuana use and were developed by treatment professionals but that neither treatment had been shown to be more effective than the other. Participants were told that assignment to the DTC condition would involve a 4-month delay, following which they would be able to choose either the RPSG or IAI treatment. They also were told that referral for more immediate treatment would be provided at any time on request. No specific instructions were given to participants in the DTC condition regarding whether to stop or continue use of marijuana during the delay period. Participants who asked were told that it was their decision.

After signing the consent form, participants completed self-report questionnaires that assessed sociodemographic characteristics, drug use, psychiatric symptoms and psychological distress, reasons for wanting to stop using marijuana, and perceived acute effects of marijuana intoxication. They were instructed to give a collateral consent packet to an adult who would be able to provide independent estimates of the participant's marijuana, alcohol, and other drug use at the follow-up assessments. The packet contained a letter of introduction and two copies of an informed consent form, one of which the collateral signed and mailed to the research office. The consent form indicated that the collateral's estimate of drug use was needed for research purposes only and that responses would not be shared with the participants.

Eligibility was determined from the initial questionnaire data. Those found ineligible were offered referral to other treatment but were not told of the criteria used to make eligibility decisions. Eligible participants returned for a second assessment session and completed additional questionnaires assessing personal goals regarding future marijuana use, self-efficacy for avoiding marijuana use, social influences on use, attitudes toward marijuana use, ways of coping with stress, and relationship with spouses or significant others, if applicable, in addition to several brief personality inventories. Once the $\$ 60$ deposit was collected and collateral consent was received, participants were randomly assigned to treatment condition and contacted regarding the start of treatment.

\section{Treatment Conditions and Procedures}

\section{RPSG}

The RPSG condition was designed to represent an extended comprehensive, outpatient treatment approach to marijuana cessation using both cognitive-behavioral and social support processes. The primary therapeutic component in this treatment condition consisted of 142 -hr group 
sessions scheduled over an 18-week period (i.e., 4 months). Sessions 1-10 were scheduled weekly, and Sessions 11-14 were scheduled every other week. The content of early sessions closely resembled prior group treatment for marijuana use based on the relapse prevention model (Stephens et al., 1994). Homework designed to encourage practice of specific skills was assigned each week and reviewed during the following session. Sessions 1-4 focused on building motivation for change by having the group list reasons for quitting and by having group discussions about perceived consequences of use and advantages of stopping use. High-risk situations for relapse were identified through self-monitoring exercises. During these sessions, participants selected either a tapered reduction or a so-called cold turkey quitting method, made public commitments to quit, sought support for quitting from others in their natural environments, and planned how to cope with high-risk situations. A quit ceremony was conducted during the fourth session of each group - the target quit date. Sessions 5-10 focused on building coping skills through planned exercises and role-plays (e.g., stress and anger management skills, how to cope with urges, self-reinforcement, and lifestyle modification). In each session, therapists facilitated discussion of participants' encounters with high-risk situations or slips into marijuana use and then used role-plays to generate alternative ways of handling similar situations in the future. In Sessions 11-14, participants were taught to identify and cope with rationalizations for returning to use and negative attributions following a slip into marijuana use. Continued attention was given to role-playing in high-risk situations and to lifestyle issues that predispose to relapse.

Starting with Session 10, a portion of each of the final four sessions included written materials and guidelines for conducting self-help sessions. Therapists conducted discussions and role-play exercises to build self-help group skills. Groups met without the therapists in the off-weeks between the 10th and 14th sessions in order to practice conducting meetings on their own. At subsequent treatment sessions, therapists debriefed the intervening self-help meetings in order to troubleshoot and facilitate the groups' continued meetings after the end of formal treatment.

RPSG participants were invited to ask a spouse, partner, relative, or close friend to attend an optional 4-session supporters' group (SG) that ran concurrently with their primary group meetings. The same therapist team that led the primary group led the corresponding SG. The SGs met during Weeks 3, 4, 5, and 11 of the 18-week treatment period. SG sessions consisted of structured activities that provided supporters with an overview and rationale for the RPSG treatment, practice in helping identify high-risk situations, and brainstorming sessions to identify alternate coping strategies. Participants were encouraged to attend SG sessions along with their supporters in order to negotiate specific relapse prevention support strategies. The final SG session, during Week 11 of the RPSG treatment, corresponded to the treatment focus on preventing relapse. 
The RPSG treatment was conducted by six master's-level therapists (three men and three women) with prior group counseling experience who were paired in male-female teams to conduct a total of 14 separate treatment groups. Each group was composed of between 8 and 12 participants. Prior to the start of treatment, therapists studied a treatment manual containing procedures for each group session, practiced leading groups in role-plays, and rehearsed specific treatment exercises under the supervision of Roger A. Roffman, a doctoral-level clinician with over 20 years experience conducting cognitive-behavioral interventions for marijuana and other drug problems. 3 Therapists met weekly as a group with the supervisor to debrief previous sessions and plan and rehearse the next sessions. Checklists of completed session activities were reviewed and discussed with the supervisor each week to facilitate treatment fidelity. SG training and supervision were conducted using similar procedures.

\section{IAI}

The IAI condition was modeled after the Drinker's Check-Up (Miller, Benefield, \& Tonigan, 1993; Miller \& Sovereign, 1989), a well-documented brief treatment that has been shown to be effective in reducing alcohol use. It consisted of two 90-min individual sessions with a therapist who provided feedback, used motivational interviewing techniques, and provided advice on cognitive-behavioral techniques that could be used to stop marijuana use. One male and one female doctoral-level clinician conducted the IAI treatment. One of the therapists, Roger A. Roffman, had attended formal training in the use of motivational interviewing techniques (Miller \& Rollnick, 1991) and served as the trainer and supervisor to promote consistency. The therapists trained together in conducting the intervention by following a structured outline for each session, role-playing and practicing motivational interviewing techniques, and debriefing completed sessions.

During the first session, the therapist reviewed a report with the participant that was generated from information collected on pretreatment assessment questionnaires. The report summarized the participant's frequency of marijuana use, problems related to use, reasons for quitting, and high-risk situations for use. Therapists also provided information on the health effects of marijuana use. Therapists in this condition used motivational interviewing techniques, such as open-ended questions, reflective listening, affirmation, and reframing, in order to elicit selfmotivational statements while avoiding confrontation (Miller \& Rollnick, 1991). Therapists then helped the participant construct a plan for quitting within the next week, complete a quit contract, and review potential antecedents to relapse. Specific high-risk situations were identified and discussed. Immediately following the first session, the therapist mailed a structured letter to the participant that reinforced commitment to change and encouraged the participant to phone 
the therapist at least once during the next month. The letter also contained a series of suggestions for coping with the high-risk situations that had been identified during the first session.

The IAI participants were invited to bring a partner or significant other to the second session, which was scheduled 1 month after the first session. During the second IAI session, the therapist reviewed marijuana use during the past month and the antecedents to use (or the temptation to use). Motivational interviewing was used to reinforce success in quitting or to rebuild motivation, as needed. A plan for sustaining (or achieving) abstinence was negotiated. If a partner or significant other was present, the therapist attempted to use the partner's comments to support motivation for abstinence and to help identify high-risk situations for relapse.

\section{Follow-Up Procedures}

Questionnaires assessing marijuana, alcohol, other drug use and related psychosocial variables were mailed to participants and collaterals 1, 4, 7, 13, and 16 months after the start of treatment. Cover letters reminded participants and collaterals that their responses were confidential and would not be shared with anyone, including therapists. Collaterals also were reminded that their responses would not be shared with the participants. Weekly phone calls and mailings were used to prompt participants and collaterals to return the questionnaires. Brief telephone interviews were conducted to collect essential outcome data if questionnaires were not returned within 2 months of the initial mailing. Overall, follow-up participation rates averaged $86 \%$ and did not differ significantly by treatment condition at any follow-up (see Table 1). Approximately 6\%, $10 \%, 17 \%, 24 \%$, and $18 \%$ of completed participant follow-ups were conducted by phone at the 1-, 4-, 7-, 13-, and 16-month follow-ups, respectively.

\section{Measures}

\section{Marijuana use}

Self-reported age of first use, age of first daily use, total years of use, and number of serious previous quit attempts were used to describe history of and chronicity of marijuana use. The selfreported number of days of marijuana use during the past 90 days, divided by 3 to yield a monthly frequency of use index, was the primary dependent measure at all time points. The number of times marijuana was used on a typical day of use in the past 90 days was assessed using a 4-point scale where $0=$ not at all, $1=$ once, $2=2-3$ times, $3=4-5$ times, and $4=6$ or more times per day. The number of uses per day was considered a proxy variable for typical quantity consumed per day. At the 1- and 4-month follow-ups, participants reported the number of days on which they used marijuana and the number of times used on a typical day of use for 
each of the 4 weeks preceding the assessment. Weekly estimates were combined to create abstinence, average frequency, and average daily use variables for the 4-week periods.

\section{Alcohol and other drug use}

Participants reported the number of times they used alcohol and other drugs during the past 90 days at baseline and the 4-, 7-, 13-, and 16-month follow-ups. The low frequency of use of drugs other than alcohol resulted in the creation of a single index of other drug use by summing across responses regarding the use of cocaine, hallucinogens, stimulants, sedatives, heroin, methadone, and other opiates during the past 90 days.

\section{Dependence symptoms and drug problems}

Severity of drug dependence was assessed by creating an 11-item marijuana dependence scale (MDS) based on the dependence criteria in the Diagnostic and Statistical Manual of Mental Disorders (3rd ed., rev.; DSM-III-R;American Psychiatric Association, 1987). The nine dependence criteria were converted directly to self-report questionnaire items with slight changes in wording to improve readability. Participants responded either yes or no to each item (e.g., "When I used marijuana, I often ended up smoking more or for longer periods of time than I intended") on the basis of whether it applied to their drug use during the past 90 days. The tolerance and withdrawal criteria each required two items in order to capture the intent of the criteria while avoiding convoluted wording. In these cases, endorsement of either or both items tapping tolerance or withdrawal phenomena counted as only one symptom in the final index. Participants answered the dependence items separately for the use of marijuana, alcohol, and all other drugs combined at the pretreatment and 4-, 7-, 13-, and 16-month assessments. Principalcomponents analyses of the scales at each assessment consistently indicated unidimensional structures. Endorsements of symptoms were counted to yield the total number of dependence criteria acknowledged for marijuana (range $=0-9$ ). 4 The internal consistency of the MDS was adequate to excellent at all assessment points (mean alpha $=.85$ ).

Participants reported problems related to the use of marijuana, alcohol, and other drugs in the past 90 days using a list of 19 negative psychological, social, occupational, and legal consequences (e.g., problems in your family, memory loss, procrastination) at the pretreatment and 4-, 7-, 13-, and 16-month assessments. The problem list was adapted from other drug use severity instruments and modified on the basis of prior research with marijuana users in treatment (Stephens et al., 1993, 1994). Participants responded to each problem on the list using a 3-point scale ranging from 0 (no problem) to 2 (serious problem). Endorsements of items on 
the list as either minor or serious problems were counted to create indices of the total number of problems (range $=0-19)$ for marijuana (mean alpha $=.90)$, alcohol (mean alpha $=.77$ ), and other drug use (mean alpha $=.92$ ).

\section{Collateral verifiers}

At each follow-up, the collateral verifiers reported on their relationships to the participants, the frequency of contact with the participants, and their confidence in the accuracy of their estimates of participants' marijuana use. Collaterals then estimated the number of days on which the participants used marijuana during the past 90 days (and for each of the past 4 weeks at the 1and 4-month follow-ups) and the number of times participants used marijuana on a typical day of use. The collaterals also reported on the participants' frequencies of use of alcohol and other drugs during the past 90 days. Problems related to drug use were assessed with the question, "In the past 90 days, do you believe the participant experienced problems in personal, social, occupational, or physical functioning because of his or her use of _," which was repeated for marijuana, alcohol, and other drugs.

\section{DTC reactions}

At the 4-month follow-up, participants in the DTC condition completed a 16-item questionnaire that assessed reactions to having to wait 4 months for treatment. Items were created to tap both negative and positive affective and motivational responses. All items were answered on a 5-point scale ranging from 1 (strongly disagree) to 5 (strongly agree). Exploratory principal-components analysis suggested three subscales with acceptable internal consistency and face validity. The Disappointment subscale was composed of seven items assessing feelings of disappointment, demoralization, discouragement, anger, and unease (alpha $=.84$ ). The Relief subscale was composed of four items related to feeling relief, not wanting to start treatment right away, and wanting the 4-month delay (alpha $=.81$ ). The Personal Responsibility subscale was composed of three items tapping feelings that being assigned to the DTC condition was "a sign that I needed to stop on my own" or that "quitting marijuana was up to me anyway" (alpha $=.54)$.

\section{Results}

\section{Pretreatment Marijuana Use, Dependence, and Problems}

Table 2 shows pretreatment history of marijuana use. The average participant used marijuana almost daily and typically used multiple times each day. Multiple quit attempts, problems related to use, and dependence symptoms were the norm. Table 3 shows the percentage of participants 
endorsing each of the dependence criteria. Only 6 participants $(2 \%)$ endorsed fewer than three symptoms, the number required for a diagnosis of dependence according to DSM-III-R (American Psychiatric Association, 1987). Participants also endorsed an average of 9.88 problems related to their marijuana use, with the most common being as follows: feeling bad about use (94\%), procrastination (94\%), reduced energy (89\%), lowered self-esteem (87\%), lowered self-confidence (79\%), and decreased productivity (74\%). Physiological problems, such as memory loss $(80 \%)$, withdrawal symptoms (66\%), and difficulty sleeping (52\%), were common as were relationship problems with partners (65\%), family (40\%), and friends (31\%). Financial difficulties (43\%) and missing work or school as a consequence of use (19\%) were reported, but legal problems were rare $(7 \%)$.

Table 2

Pretreatment Marijuana Use

\begin{tabular}{lrr}
\hline \multicolumn{1}{c}{ Variable } & \multicolumn{1}{c}{$M$} & \multicolumn{1}{c}{$S D$} \\
\hline Age of first use & 15.93 & 3.90 \\
Age of first daily use & 19.60 & 5.60 \\
Years of use & 17.35 & 5.21 \\
No. of previous quit attempts & 3.94 & 21.97 \\
Days of use, past 90 days & 74.64 & 18.54 \\
Times used per day, past 90 days & 2.54 & 0.89 \\
No. of dependence symptoms & 6.74 & 1.97 \\
No. of marijuana-related problems & 9.88 & 2.97 \\
\hline
\end{tabular}

Note. $\quad N=291$.

${ }^{a} 1=$ once, $2=2-3$ times, $3=4-5$ times, $4=6$ or more times.

Table 3

Pretreatment Endorsement of DSM-III-R Dependence Criteria

\begin{tabular}{lc}
\hline \multicolumn{1}{c}{ Dependence symptom } & Percentage endorsing \\
\hline Continued using despite knowing it contributed to problems & 93 \\
Tried unsuccessfully to cut down or control use & 90 \\
Frequently high or recovering when attending to obligations or & 86 \\
engaging in hazardous activities & 79 \\
Experienced withdrawal symptoms when trying to stop use & 75 \\
Spent great deal of time getting, using, or recovering & 69 \\
Used more or for longer periods than intended & 63 \\
Tolerance: needed to use more to get the same effects or did not & 61 \\
get as high when smoking the same amount as in the past & 58 \\
Often used to relieve or avoid withdrawal symptoms & \\
Gave up or did not participate in important activities because of use & \\
\hline
\end{tabular}

Note. $\quad N=291$. DSM-III-R = Diagnostic and Statistical Manual of Mental Disorders (3rd ed., rev.; American Psychiatric Association, 1987).

\section{Treatment Participation and Fidelity}


The average number of RPSG treatment sessions attended was $8.42(\mathrm{SD}=3.51)$ out of a possible 14. Fifty percent of RPSG participants attended 10 or more sessions. Forty-six RPSG participants (39\%) had a supporter who attended at least one of the four SG sessions, and these supporters attended an average of 2.46 ( $\mathrm{SD}=1.11$ ) of the four SG sessions. Seventy-six of the 88 IAI participants $(86 \%)$ attended both sessions. A supporter accompanied 31 IAI participants $(35 \%)$ at the second session. The percentages of participants who reported attending additional formal treatment (3\%-7\% across follow-ups) or self-help groups (8\%-15\% across follow-ups) for drug-related problems (e.g., Marijuana Anonymous, Narcotics Anonymous) were small and did not vary significantly by treatment condition at any follow-up. At the 4-month follow-up, $6 \%$ of the DTC participants reported attending formal treatment during the waiting period, and $18 \%$ reported attending self-help groups. However, these rates of outside treatment attendance did not differ significantly from those reported by participants in the IAI and RPSG conditions (i.e., formal treatment $=3 \%$; self-help groups $=13 \%$ ).

The average RPSG participant attended 1.62 (SD = 1.45) of the four scheduled self-help meetings that alternated with the final four therapist-led sessions. Analyses of self-help meeting attendance across the 14 RPSG treatment groups revealed substantial variability. At the 7-month follow-up, 36\% of participants reported attending at least one meeting of their self-help groups after the end of formal treatment. Comparable attendance rates for the period since the previous assessment were $23 \%$ and $8 \%$ at the 13- and 16-month follow-ups, respectively. Although 8 of the 14 groups continued meeting during the first 3 months after treatment, only 4 were still meeting at the 13-month follow-up and 2 were still meeting as of the 16-month follow-up.

Participants' ratings of RPSG and IAI therapists on 7-point bipolar adjective scales at the 1month follow-up showed that they were generally perceived positively by participants. Means ranged from 5.30 to 6.60 , with higher scores indicative of more positive attributes. Significant differences between treatment conditions were detected on 3 of 12 adjective scales, ps $<.05$. The IAI therapists were rated as more caring and less active than the RPSG therapist teams, differences that are consistent with the empathic, personalized, and less directive style of motivational interviewing (Miller \& Rollnick, 1991). IAI therapists $(M=6.45)$ also were rated as significantly more competent than the RPSG therapists $(M=6.00)$, although means for both conditions approached the competent end of the scale. RPSG and IAI therapists were not rated significantly different on scales assessing optimism, warmth, helpfulness, sociability, sensitivity, enthusiasm, friendliness, interest, and nurturance.

Participants' ratings of treatment on 7-point scales ranging from 1 (not at all helpful) to 7 (extremely helpful) showed that the RPSG treatment was perceived to be more helpful at the 1- 
and 4-month follow-up assessment points ( $\mathrm{Ms}=5.57$ and 5.36, respectively) than the IAI treatment $(\mathrm{Ms}=5.04$ and 4.50 , respectively), $\mathrm{ps}<.01$. Participants' ratings of the occurrence and helpfulness of treatment-specific activities converged with therapist checklist data in demonstrating that planned activities almost always occurred in both treatment conditions (i.e., greater than $95 \%$ of the time) and indicated that all activities were perceived as at least moderately helpful.

\section{Marijuana, Alcohol, and Other Drug Outcomes}

Prior to examining outcomes, we compared the characteristics of participants who completed and who did not complete follow-ups using two-way (Treatment Condition $\times$ Follow-Up Completion) analysis of variance in order to detect bias in the follow-up samples. We performed these analyses separately on 19 pretreatment drug use and sociodemographic variables reported in this article and repeated them five times, using the follow-up completion status of the participants at each of the five follow-ups. Of the 285 significance tests ( 19 variables $\times 5$ followups $\times 3$ effects), only five $(<2 \%)$ reached significance at $\mathrm{p}<.05$. The few significant findings were small in magnitude and spread across several variables without a consistent pattern. Thus, the follow-up samples used in the outcome analyses appeared to be representative of the randomized sample.

\section{Validity of self-reports}

Collateral verification of marijuana, alcohol, and other drug use was obtained for an average of $85 \%$ of the participants who completed follow-up assessments. Collaterals were spouses or partners $(54 \%)$, other relatives (11\%), or friends (35\%) of the participants. Most collaterals $(58 \%)$ reported seeing the participant almost every day during the assessment period. A smaller proportion of collaterals saw the participant less than once per week $(15 \%)$ or had only phone or mail contact $(5 \%)$ during the assessment period. Collaterals' confidence in the accuracy of their reports of participants' drug use averaged 5.45 on a scale ranging from 1 (not at all confident) to 7 (extremely confident). 5

The average correlations between participant and collateral reports of days of marijuana use and number of times used on a typical day of use were .75 and .66 , respectively. Agreement on complete abstinence for the assessment period averaged $87 \%$ across follow-ups. Agreement on the existence of marijuana-related problems averaged $62 \%$; however, only $4 \%$ of the disagreements occurred because the collateral reported problems when the participant did not. In 
general, for every index of marijuana use, the vast majority of discrepancies occurred because participants reported more use than collaterals.

The average correlation between participant and collateral reports of frequency of alcohol use was .69 , and agreement on the occurrence of alcohol-related problems averaged $73 \%$. The average correlation between collateral and participant reports of the frequency of other drug use was .28. This correlation appeared to be suppressed by the large number of participants at each follow-up (75\%-85\%) who did not report any other drug use, thus skewing the distributions, restricting the range, and allowing a few cases to be overly influential. As was typical with the alcohol use indices, only $5 \%$ of the discrepancies on drug use were the result of a collateral estimating more use than the participant, and agreement was consistently high (92\%) for identification of problems related to other drug use. These data suggest that participants did not systematically underreport their use of marijuana, alcohol, or other drugs.

\section{Marijuana outcome comparisons involving the DTC condition}

General linear model (GLM) analyses were performed separately on measures of marijuana use, dependence symptoms, and marijuana-related problems during the past 90 days with treatment condition (RPSG, IAI, DTC) as the between-participants factor and time (pretreatment assessment vs. 4-month follow-up) as the within-participants factor. Main effects of treatment and time on every measure (all ps $<.01$ ) were qualified by significant Treatment $\times$ Time interactions for days of use per month, $\mathrm{F}(2,245)=23.23, \mathrm{p}<.001, \eta 2=.16$; times used per day, $\mathrm{F}(2,245)=9.92, \mathrm{p}<.001, \eta 2=.08$; marijuana problems, $\mathrm{F}(2,230)=24.62, \mathrm{p}<.001, \eta 2=.18$; and dependence symptoms, $\mathrm{F}(2,220)=21.34, \mathrm{p}<.001, \eta 2=.16 .6$ There were no significant differences between treatment conditions on any measure at the pretreatment assessment. Participants in all three treatment conditions reported statistically significant reductions in marijuana use and related consequences on all measures at the 4-month follow-up, all ps $<.001$ (see Table 4). RPSG and IAI participants reported fewer days of use, times of use per day, problems related to marijuana, and dependence symptoms than DTC participants at the 4-month follow-up, all ps <.001. RPSG and IAI participants, however, did not differ significantly on any indicator of use or associated problems. 7 
Table 4

Marijuana Use, Dependence Symptoms, and Related Problems During the Past 90 Days

\begin{tabular}{|c|c|c|c|c|c|c|c|c|c|c|}
\hline \multirow{3}{*}{$\begin{array}{l}\text { Treatment } \\
\text { condition }\end{array}$} & \multicolumn{10}{|c|}{ Assessment } \\
\hline & \multicolumn{2}{|c|}{ Pretreatment } & \multicolumn{2}{|c|}{4 months } & \multicolumn{2}{|c|}{7 months } & \multicolumn{2}{|c|}{13 months } & \multicolumn{2}{|c|}{16 months } \\
\hline & $M$ & $S D$ & $M$ & $S D$ & $M$ & $S D$ & $M$ & $S D$ & $M$ & $S D$ \\
\hline \multicolumn{11}{|c|}{ Days of use per month } \\
\hline RPSG & $25.38_{a}$ & 6.15 & $6.68_{\mathrm{a}}$ & 9.87 & $8.999_{a}$ & 10.87 & $11.89 \mathrm{a}$ & 12.10 & $12.29 \mathrm{a}$ & 12.34 \\
\hline IAI & $24.24_{\mathrm{a}}$ & 6.29 & $7.88_{\mathrm{a}}$ & 10.98 & $8.50_{a}$ & 10.82 & $11.6 \mathrm{I}_{\mathrm{a}}$ & 11.44 & 12.99 & 11.61 \\
\hline DTC & $24.85_{\mathrm{a}}$ & 6.13 & $17.09_{\mathrm{b}}$ & 10.73 & 一 & 一 & 一 & 一 & 一 & 一 \\
\hline \multicolumn{11}{|c|}{ Times used per day ${ }^{\mathrm{a}}$} \\
\hline RPSG & $2.59_{\mathrm{a}}$ & 0.89 & $1.15_{\mathrm{a}}$ & 1.10 & $1.3 \mathrm{I}_{\mathrm{a}}$ & 1.11 & $1.52_{\mathrm{a}}$ & 1.21 & $1.39 \mathrm{a}$ & 1.15 \\
\hline IAI & $2.41_{\mathrm{a}}$ & 0.85 & $1.19_{\mathrm{a}}$ & 1.18 & $1.10_{a}$ & 1.14 & $1.3 \mathrm{I}_{\mathrm{a}}$ & 1.10 & $1.41_{\mathrm{a}}$ & 1.20 \\
\hline DTC & $2.6 \mathrm{I}_{\mathrm{a}}$ & 0.93 & $1.97_{b}$ & 1.09 & 一 & 一 & 一 & 一 & 一 & 一 \\
\hline \multicolumn{11}{|c|}{ No. of dependence symptoms } \\
\hline RPSG & $6.84_{\mathrm{a}}$ & 2.13 & $1.96 \mathrm{a}$ & 2.73 & $2.52_{a}$ & 3.02 & $2.67_{\mathrm{a}}$ & 3.13 & $2.83 a$ & 3.27 \\
\hline IAI & $6.65_{\mathrm{a}}$ & 1.95 & $1.94_{\mathrm{a}}$ & 2.71 & $2.05_{a}$ & 2.57 & $2.66_{\mathrm{a}}$ & 2.92 & $2.75_{\mathrm{a}}$ & 3.18 \\
\hline DTC & $6.71_{\mathrm{a}}$ & 1.77 & $4.63_{b}$ & 2.59 & 一 & 一 & 一 & 一 & 一 & 一 \\
\hline \multicolumn{11}{|c|}{ No. of marijuana-related problems } \\
\hline RPSG & $9.86_{\mathrm{a}}$ & 3.05 & $3.50_{\mathrm{a}}$ & 4.23 & 4.29 & 4.66 & $4.14_{\mathrm{a}}$ & 4.60 & $4.21_{\mathrm{a}}$ & 4.98 \\
\hline IAI & $9.99_{\mathrm{a}}$ & 2.89 & $3.26_{a}$ & 3.99 & $4.02_{a}$ & 4.70 & $4.62_{a}$ & 4.70 & $4.71_{\mathrm{a}}$ & 4.74 \\
\hline DTC & $9.78_{a}$ & 2.96 & $7.89_{b}$ & 4.23 & - & - & - & - & - & - \\
\hline
\end{tabular}

Note. Pretreatment data are presented for the complete sample $(N=291)$. Sample sizes vary at follow-up (see Table $I$ and text). Means for all treatment conditions on all variables are reduced significantly at every follow-up relative to the corresponding pretreatment value, $p<.001$. At a given assessment, means with different subscripts for the same variable are significantly different at $p<.001$. RPSG $=$ relapse prevention support group; $\mathrm{IAI}=$ individualized assessment and advice; DTC $=$ delayed treatment control. Dashes indicate that DTC participants were not assessed at these follow-ups.

${ }^{a} 1=$ once, $2=2-3$ times, $3=4-5$ times, $4=6$ or more times.

Abstinence rates for the 90 days prior to the 4-month assessment were nearly identical for the RPSG and IAI conditions (37\%) and significantly greater than for the DTC condition (9\%), $\chi 2(\mathrm{~N}$ $=248)=21.46, p<.001, \varphi=.29$. In a similar manner, RPSG (44\%) and IAI (39\%) participants reported higher rates of abstinence than DTC participants (17\%) for the 4 weeks immediately prior to the 4-month follow-up, $\chi 2(\mathrm{~N}=243)=14.74, \mathrm{p}<.001, \varphi=.25$.

\section{DTC reactions and treatment participation}

Mean ratings on the 5-point scales assessing DTC participants' reactions to being assigned to wait for treatment were $2.75(\mathrm{SD}=0.78)$ for disappointment, $2.47(\mathrm{SD}=0.88)$ for relief, and 3.00 ( $\mathrm{SD}=0.68$ ) for personal responsibility. In order to assess the relationship between reactions to DTC assignment and changes in marijuana use during the 4-month waiting period, we computed partial correlations between each of the subscales and marijuana use at the 4-month 
follow-up after controlling for the corresponding marijuana use variable at pretreatment. The Personal Responsibility subscale was significantly related to both frequency of marijuana use ( $\mathrm{r}$ $=-.62, \mathrm{p}<.001)$ and the number of uses on a typical day $(\mathrm{r}=-.43, \mathrm{p}<.001)$ after controlling for pretreatment use. The more participants endorsed items indicating that change in marijuana was their own responsibility, the less frequently they used marijuana during the waiting period. The Disappointment and Relief subscales were not related significantly to change in marijuana use during the waiting period.

\section{Marijuana outcome comparisons involving only RPSG and IAI conditions}

At the 1-month follow-up, we compared treatment conditions using two condition (RPSG vs. IAI) analyses of covariance (ANCOVAs) because of the lack of comparable week-by-week marijuana use indices at pretreatment. The covariate in each analysis was the corresponding measure assessed for the full 90-day pretreatment window. At the 1-month follow-up, IAI participants $(\mathrm{M}=1.60 ; \mathrm{SD}=2.07)$ reported using marijuana significantly fewer days per week than RPSG participants $(\mathrm{M}=2.49$; $\mathrm{SD}=2.32), \mathrm{F}(1,177)=5.63, \mathrm{p}<.02, \eta 2=.03$. IAI participants also reported using fewer times per day $(\mathrm{M}=0.89 ; \mathrm{SD}=1.43)$ than $\mathrm{RPSG}$ participants $(\mathrm{M}=2.00 ; \mathrm{SD}=2.98), \mathrm{F}(1,176)=6.72, \mathrm{p}<.01, \eta 2=.04$. Abstinence rates for the past 4 weeks at the 1-month follow-up were significantly higher in the IAI (42\%) than RPSG conditions $(27 \%), \chi 2(\mathrm{~N}=180)=4.30, \mathrm{p}<.04, \varphi=.16$.

In order to examine longer-term outcomes, we used marijuana use, dependence symptom, and problem indices assessed for the past 90 days as dependent variables in a series of 2 (treatment) $\times 2$ (time) GLM analyses. Treatment condition (RPSG vs. IAI) was the between-participants factor, and time was the within-participants factor created by comparing pretreatment indices with the corresponding indices at each follow-up. Significant effects of time were found in each analysis, indicating that marijuana use and associated consequences were reduced at each followup relative to pretreatment levels, all ps $<.001$ (see Table 4). There were no significant Treatment $\times$ Time interactions indicative of differential treatment outcomes. A single main effect of treatment condition in the analysis of 7-month follow-up data indicated that RPSG participants reported using marijuana more times per day than IAI participants. However, when ANCOVA was used to control for typical daily use at pretreatment, there was no difference between treatment conditions in the 7-month follow-up data.

Rates of abstinence for the past 90 days at the 7-, 13-, and 16-month follow-ups did not differ significantly between the RPSG (32\%, 26\%, and 29\%, respectively) and IAI $(36 \%, 28 \%$, and 
$28 \%$, respectively) conditions. Overall, $22 \%$ of participants reported no use of marijuana at any of these follow-ups, with no differences between treatments.

\section{Changes in alcohol and other drug use}

Many drug abuse treatment programs stress abstinence from all psychoactive substances because of a fear that participants who decrease their use of one drug will substitute another. The RPSG and IAI treatments were designed to produce abstinence from marijuana but did not directly address the use of alcohol or other drugs. In order to explore whether posttreatment decreases in marijuana use were accompanied by increases in alcohol or other drug use, we performed a parallel series of GLM analyses on the self-reported frequency of alcohol and other drug use and related problems. Treatment condition was the between-participants factor, and time (pretreatment vs. each follow-up) was the within-participants factor. No significant effects of treatment condition, time, or the interaction of treatment and time were found on the frequency of alcohol use measures. Analyses of other drug use revealed a Treatment $\times$ Time interaction at the 4-month follow-up, $\mathrm{F}(2,215)=4.59, \mathrm{p}<.05, \eta 2=.04$. Other drug use did not differ between treatment conditions at pretreatment, but DTC participants were using other drugs more frequently $(\mathrm{M}=5.01)$ at the 4-month follow-up than RPSG $(\mathrm{M}=0.76)$ or IAI $(\mathrm{M}=0.48)$ participants, $\mathrm{ps}<.05$. There were no other significant effects of time, treatment, or their interaction at the remaining follow-ups. Similar analyses revealed significant increases in the number of alcohol problems at every follow-up (all ps <.05), and increases in other drug problems were significant at the 7-month follow-up $(p<.01)$. However, there were no significant effects of treatment or the Treatment $\times$ Time interactions for alcohol or other drug-related problems at any follow-up. Means and standard deviations for alcohol and other drug use measures are presented in Table 5 collapsed across treatment condition because of the general lack of significant effects of treatment.

Table 5

Alcohol and Other Drug Use and Problems During the Past 90 Days

\begin{tabular}{|c|c|c|c|c|c|c|c|c|c|c|}
\hline \multirow[b]{3}{*}{ Variable } & \multicolumn{10}{|c|}{ Assessment } \\
\hline & \multicolumn{2}{|c|}{ Pretreatment } & \multicolumn{2}{|c|}{4 months } & \multicolumn{2}{|c|}{7 months } & \multicolumn{2}{|c|}{13 months } & \multicolumn{2}{|c|}{16 months } \\
\hline & $M$ & $S D$ & $M$ & $S D$ & $M$ & $S D$ & $M$ & $S D$ & $M$ & $S D$ \\
\hline Frequency of alcohol use & 18.06 & 22.31 & 21.30 & 25.29 & 21.34 & 27.51 & 24.93 & 30.24 & 24.84 & 30.16 \\
\hline Frequency of other drug use & 1.65 & 6.67 & 2.46 & 12.18 & 1.43 & 7.30 & 1.48 & 7.98 & 3.30 & 19.64 \\
\hline No. of alcohol problems & 0.38 & 0.95 & $0.81_{\text {a }}$ & 1.72 & $0.86_{\mathrm{a}}$ & 2.06 & $0.95_{\mathrm{a}}$ & 1.80 & $0.91_{a}$ & 1.97 \\
\hline No. of other drug problems & 0.17 & 1.12 & 0.28 & 1.31 & $0.48_{a}$ & 1.82 & 0.34 & 1.38 & 0.31 & 1.49 \\
\hline
\end{tabular}


Although the data in Table 5 suggest only modest increases in the alcohol and other drug measures, we explored whether these changes were systematically related to the frequency of posttreatment marijuana use. We computed partial correlation coefficients between the frequency of marijuana use and the measures of alcohol and other drug use at each follow-up, controlling for the corresponding pretreatment measures. There were no significant relationships between the frequency of marijuana use and the measures of other drug use, other drug problems, or alcohol problems at any follow-up. The frequency of alcohol use was correlated with the frequency of marijuana use at the 4-month $(\mathrm{r}=.18)$ and 13-month $(\mathrm{r}=.25)$ follow-ups $(\mathrm{ps}<.01)$, after controlling for frequency of pretreatment marijuana and alcohol use. Contrary to a drug substitution effect, these modest positive relationships indicated, if anything, that reductions in marijuana use were associated with reductions in alcohol use.

\section{Outcomes for treatment completers}

To explore whether the similar outcomes for the RPSG and IAI interventions were related to the lower treatment completion rate in the RPSG condition, we repeated the primary outcome analyses for treatment completers only. Treatment completion was defined as attending at least 10 of the 14 therapist-led RPSG sessions $(n=58)$ or both IAI sessions $(n=76)$. It should be noted that the RPSG treatment completers were much more highly selected and comprised only $50 \%$ of the randomized sample, whereas $86 \%$ of the IAI participants were treatment completers. Outcomes on each dependent variable were examined using two-condition (RPSG vs. IAI) ANCOVAs with the corresponding pretreatment variable as the covariate. At the 4-month follow-up, RPSG treatment completers were using marijuana fewer days per month $(\mathrm{M}=3.12$; $\mathrm{SD}=5.89)$ than IAI treatment completers $(\mathrm{M}=6.77 ; \mathrm{SD}=10.07), \mathrm{F}(1,118)=7.02, \mathrm{p}<.01, \eta 2$ $=.06$. However, there were no significant differences between conditions on days of use at the 7 , 13-, or 16-month follow-ups. Furthermore, IAI and RPSG treatment completers did not differ on measures of use per day, dependence symptoms, or problems at any follow-up.

\section{Discussion}

This project compared two interventions of substantially different intensities in a controlled treatment-outcome study of adults seeking treatment for marijuana use. It is noteworthy that this is only the second controlled trial to focus on the treatment of marijuana use disorders, although others are in progress (e.g., Budney, Radonovich, Higgins, \& Wong, 1998). The dearth of data on effective treatments is noteworthy in light of recent data on the extent of marijuana dependence and the potential health concerns. It is notable that both an extended group intervention and a brief individual intervention produced substantial reductions in marijuana use and related problems relative to the delayed treatment condition. However, there were few 
differences in the outcomes for the RPSG and IAI interventions, suggesting that the brief individual treatment was just as effective as the more extended group therapy for this population.

Both the RPSG and IAI treatments resulted in substantial reductions in marijuana use, dependence symptoms, and related problems at the 4-month follow-up relative to the DTC condition. RPSG and IAI participants were using marijuana at approximately $30 \%$ of their pretreatment rate 4 months after the initiation of treatment, whereas DTC participants reduced their use to approximately $70 \%$ of pretreatment frequency. Relative reductions of similar magnitude were found for intensity of use per day, dependence symptoms, and problems related to use. Between-condition effect sizes (d; Cohen, 1988) relative to the DTC condition were in the range of $0.70-1.10$ on these measures for both interventions, representing relatively large effects.

This is one of the first trials of drug abuse treatments to include a delayed treatment control condition in order to control for the effects of pretreatment assessment and participants' motivation for change or other maturational processes within the participants. DTC participants reduced their marijuana use significantly during the waiting period but to a much smaller degree than those participating in the RPSG and IAI treatments. It is possible, of course, that the DTC condition did not accurately represent the natural course of marijuana use in the absence of treatment. Attendance of treatment outside of the study may have accounted for some of the observed change, but rates of other treatment attendance were low and did not differ by treatment condition. Assignment to the DTC condition also may have been perceived as permission to continue using marijuana or even may have created demand characteristics that participants should not make changes. Disappointment associated with having to wait for treatment might have contributed to poorer outcomes for these participants. However, retrospective ratings of participants' reactions to DTC assignment suggested that those DTC participants who saw change in marijuana use as their personal responsibility were likely to reduce use in the absence of treatment. Feelings of disappointment or relief at not having to start treatment were not systematically related to changes in marijuana use. Thus, the DTC condition appeared to capture the likely course of marijuana use for treatment seekers in the absence of intervention. The greater reductions in marijuana use in the RPSG and IAI conditions, therefore, cannot be attributed solely to participants' motivation or reactivity to pretreatment assessment.

Contrary to our predictions, RPSG participants did not achieve greater reductions in marijuana use than IAI participants. In fact, IAI participants reduced their marijuana use more during the first month of treatment; a finding that may be related to the difference in quitting promoted by the interventions. RPSG participants spent the first 4 weeks of treatment learning to identify high-risk situations for use and preparing to quit, whereas IAI participants developed plans for 
quitting during the first session. There were no differences between RPSG and IAI treatments in marijuana use, dependence symptoms, or problems related to use at subsequent follow-ups. Participants in both treatments were using less than half as many days at the 16-month follow-up as they were before treatment. Almost $30 \%$ of participants in both treatment conditions were completely abstinent for the 90 days preceding the 16-month follow-up, and $22 \%$ sustained abstinence throughout the last 12 months of the follow-up period. These reductions in marijuana use were achieved without related increases in alcohol or other drug use.

The lack of differences in outcomes between the RPSG and IAI conditions at the 4-month follow-up was particularly surprising because RPSG participants had just completed treatment, whereas IAI participants had completed treatment 3 months earlier. When only treatment completers were examined at the 4-month follow-up, the RPSG participants showed greater reductions in the frequency of marijuana use than the IAI participants, but this advantage was lost at later follow-ups. Furthermore, RPSG and IAI treatment completers did not differ on indices of typical daily use, problems, or dependence symptoms related to use at any follow-up. These post hoc comparisons of treatment completers likely were biased in favor of the RPSG intervention. Treatment completion required greater commitment to treatment for RPSG than IAI participants, and dropping out of group treatment has been associated with sociodemographic and psychological variables that predict poor marijuana use outcomes (Roffman, Klepsch, Wertz, Simpson, \& Stephens, 1993). Thus, even when controlling for exposure to the full intervention, the extended RPSG treatment did not produce outcomes superior to the brief IAI treatment.

Although we did not calculate the relative costs of delivering the brief versus extended treatments, the results suggest that brief treatments may be more cost-effective for some adult marijuana users. Two sessions of individualized feedback and advice on how to quit produced reductions in marijuana use comparable to that of a 14-session group intervention, with additional sessions for supporters and ongoing self-help meetings. The greater experience and qualifications of the IAI therapists in comparison with the RPSG therapists in this study may have increased costs somewhat for the brief treatment, but other research suggests that even novice therapists can deliver similar treatments with success (e.g., Miller et al., 1993). Studies are needed to examine systematically the relative costs of providing brief versus extended treatments in relation to the degree of reductions in drug use and related consequences. It is worth noting that $64 \%$ of the 64 DTC participants who subsequently participated in treatment chose the IAI condition, suggesting that brief treatments are appealing.

It is unfortunate that our data can say little about the treatment processes that produced change in marijuana use and related symptoms. Close attention was paid to treatment fidelity during the 
supervision of therapists, and participant ratings confirmed both the perceived quality of the therapists and the delivery of treatment-specific exercises. However, we did not audiotape and code therapist-participant interactions, nor did we measure changes in potential mediating variables (e.g., coping skills, motivation for change, social support). Both treatments attempted to solidify motivation for change early in the therapy process, albeit by different mechanisms given the group versus individual modalities. Both treatments provided cognitive-behavioral techniques for avoiding marijuana use. It is possible that these were the active ingredients in both interventions. The comparable outcomes then would suggest that added rehearsal of coping skills, attention to lifestyle issues, education about cognitive precipitants of relapse, and the adjunctive social support interventions in the RPSG condition did not add to these basic components. It must be noted, however, that the attempt to build ongoing support groups from the therapy groups had limited success, with few groups continuing to meet after treatment ended. Future research must measure the processes within treatment sessions and other potential mediating variables more carefully in order to relate them to outcomes.

Characteristics of participants in the current study and treatment outcomes were strikingly similar to our prior study (Stephens et al., 1993, 1994). The sample was composed largely of employed, white men in their early 30s who were daily marijuana smokers for 10 or more years. Most participants appeared to meet diagnostic criteria for cannabis dependence, and multiple problems related to marijuana use were endorsed. However, the absence of individual interviews with participants prohibited the use of structured diagnostic instruments for cannabis dependence or abuse, thus limiting the confidence with which formal diagnoses can be attributed. Nevertheless, the MDS had good internal consistency and high face validity in relation to DSMIII-R diagnostic criteria. The Marijuana-Related Problems scale borrowed from instruments created for the assessment of alcohol and generic drug problems. We believe that the consistent pattern of findings across measures of use and related consequences and the high levels of agreement with collateral reports indicate that these measures capture abuse and dependence phenomena of clinical import. Nevertheless, studies must be conducted to validate these instruments in relation to structured interviews and other psychometrically sound assessments. Replication of the present findings with validated diagnostic and drug severity instruments is needed to confirm the initial prevalence and subsequent change in diagnosable disorders in this population of marijuana users.

Generalization of the present findings may be limited to treatment-seeking marijuana users with similar sociodemographic characteristics. Attempts to attract more minority participants through specific media were relatively unsuccessful and may partially reflect a lower prevalence of other drug dependence in non-White racial groups (Anthony et al., 1994). In a similar manner, the disproportionate representation of men is consistent with epidemiological studies showing that 
the prevalence of cannabis dependence is approximately three times as great in men as in women (Anthony et al., 1994). Thus, the effectiveness of these interventions for participants with more diverse sociodemographic characteristics is unknown, and it remains possible that greater differences may be found between the RPSG and IAI treatments in other populations. Additional studies are needed to address the generalizability of the findings across diverse client populations and to examine the possibility of matching clients to appropriate treatments on the basis of individual characteristics. Studies that directly measure individual differences hypothesized to moderate responses to brief versus extended treatments (e.g., readiness to change) are needed to help guide cost-effective treatment planning.

\section{Footnotes}

1 Participants were ineligible because of alcohol or other drug abuse if they reported three or more dependence symptoms (American Psychiatric Association, 1987) associated with their recent use of alcohol or illicit drugs or reported a pattern of problems in the past 90 days that was indicative of abuse.

2 Sequential eligible participants were accumulated into pools of between 20 and 30 participants and then randomly assigned to the three conditions after blocking on gender. The number of participants assigned to RPSG is greater because it was always necessary to assign at least 8-10 participants to RPSG in order to start a group.

3 Copies of treatment manuals are available from Roger A. Roffman, School of Social Work, University of Washington, 4101 15th Avenue N. D., Seattle, Washington 98105. Electronic mail may be sent to roffman@u.washington.edu.

4 The alcohol and other drug dependence scales were used primarily to help identify and exclude participants who were showing signs of dependence on these drugs. Outcome analyses are not presented on these scales because of the low frequency of symptom endorsement.

5 Although collaterals were instructed to provide independent estimates of participant drug use and related problems, they likely varied in the extent to which they relied on direct observation of use, secondary signs (e.g., odor, behavior changes), and the verbal reports of the participants regarding their own use. Thus, collateral reports do not represent a gold standard against which the participants' self-reports can be compared to establish validity. However, agreement between 
collaterals and participants is supportive of the conclusion that self-reports are valid. The participant's awareness of the collateral's verifying role in the study also was likely to encourage accurate reporting by participants (see Babor, Stephens, \& Marlatt, 1987).

6 Sample size is reduced in analyses of dependence symptoms and marijuana problems because these data were not available for participants assessed with brief telephone interviews and because of missing data within scales.

7 Results are the same when analyses of covariance are conducted on the 4-month follow-up indices by treatment condition with the corresponding pretreatment indices used as the covariates.

\section{References}

American Psychiatric Association. (1987). Diagnostic and statistical manual of mental disorders (3rd ed., rev.). Washington, DC: Author.

Anthony, J. C., \& Helzer, J. E. (1991). Syndromes of drug abuse and dependence. In L. N.Robins \& D. A.Regier (Eds.), Psychiatric disorders in America (pp. 116-154). New York: Free Press.

Anthony, J. C., Warner, L. A., \& Kessler, R. C. (1994). Comparative epidemiology of dependence on tobacco, alcohol, controlled substances, and inhalants: Basic findings from the national comorbidity survey. Experimental and Clinical Psychopharmacology, 2, 244-268.

Babor, T. F., Stephens, R. S., \& Marlatt, G. A. (1987). Verbal report methods in clinical research on alcoholism: Response bias and its minimization. Journal of Studies on Alcohol, 48, 410-424.

Bien, T. H., Miller, W. R., \& Tonigan, S. (1993). Brief interventions for alcohol problems: A review. Addiction, 88, 315-336. 
Budney, A. J., Radonovich, K. J., Higgins, S. T., \& Wong, C. J. (1998). Adults seeking treatment for marijuana dependence: A comparison to cocaine-dependent treatment seekers. Experimental and Clinical Psychopharmacology, 6, 1-8.

Cohen, J. (1988). Statistical power analysis for the behavioral sciences (2nd ed.). Hillsdale, NJ: Erlbaum.

Day, N. L., Richardson, G. A., Goldschmidt, L., Robles, N., Taylor, P. M., Stoffer, D. S., Cornelius, M. D., \& Geva, D. (1994). Effect of prenatal marijuana exposure on the cognitive development of offspring at age three. Neurotoxicology and Teratology, 16, 169-175.

Fleming, M. F., Barry, K. L., Manwell, L. B., Johnson, K., \& London, R. (1997). Brief physician advice for problem drinkers. Journal of the American Medical Association, 277, 1039-1045.

Fried, P. A. (1995). The Ottawa Prenatal Prospective Study (OPPS): Methodological issues and findings: It's easy to throw the baby out with the bath water. Life Sciences, 56, 2159-2168.

Haas, A. P., \& Hendin, H. (1987). The meaning of chronic marijuana use among adults: A psychosocial perspective. Journal of Drug Issues, 17, 333-348.

Hall, W., Solowij, N., \& Lemon, J. (1994). The health and psychological consequences of cannabis use. (National Drug Strategy Monograph Series No. 25). Canberra, Australia: Australian Government Publishing Service.

Marlatt, G. A., \& Gordon, J. R. (1985). Relapse prevention: Maintenance strategies in the treatment of addictive behaviors. New York: Guilford Press.

McCrady, B. S. (1989). Extending relapse prevention models to couples. Addictive Behaviors, $14,69-74$. 
Miller, W. R., Benefield, G. S., \& Tonigan, J. S. (1993). Enhancing motivation for change in problem drinking: A controlled comparison of two therapist styles. Journal of Consulting and Clinical Psychology, 61, 455-461.

Miller, W. R., \& Rollnick, S. (1991). Motivational interviewing: Preparing people to change addictive behavior. New York: Guilford Press.

Miller, W. R., \& Sovereign, R. G. (1989). The check-up: A model for early intervention in addictive behaviors. In T.Loberg, W. R.Miller, P. E.Nathan, \& G. A.Marlatt (Eds.), Addictive behaviors: Prevention and early intervention (pp. 219-231). Amsterdam: Swets \& Zeitlinger.

O'Farrell, T. J., Choquette, K. A., Cutter, H. S. G., Brown, E. D., \& McCourt, W. F. (1993). Behavioral marital therapy with and without additional couples relapse prevention sessions for alcoholics and their wives. Journal of Studies on Alcohol, 54, 652-666.

Pope, H. G., \& Yurgelun-Todd, D. (1996). The residual cognitive effects of heavy marijuana use in college students. Journal of the American Medical Association, 275, 521-527.

Rainone, G. A., Deren, S., Kleinman, P. H., \& Wish, E. D. (1987). Heavy marijuana users not in treatment: The continuing search for the "pure" marijuana user. Journal of Psychoactive Drugs, $19,353-359$.

Richardson, G. A., Day, N. L., \& Goldschmidt, L. (1995). Prenatal alcohol, marijuana, and tobacco use: Infant mental and motor development. Neurotoxicology and Teratology, 17, 479487.

Roffman, R. A., Klepsch, R., Wertz, J. S., Simpson, E. E., \& Stephens, R. S. (1993). Predictors of attrition from an outpatient marijuana-dependence counseling program. Addictive Behaviors, 18, 553-566. 
Solowij, N., Michie, P. T., \& Fox, A. M. (1991). Effects of long-term cannabis use on selective attention: An event-related potential study. Pharmacology Biochemistry and Behavior, 40, 683688.

Solowij, N., Michie, P. T., \& Fox, A. M. (1995). Differential impairments of selective attention due to frequency and duration of cannabis use. Society of Biological Psychiatry, 37, 731-739.

Stephens, R. S. (1999). Cannabis and hallucinogens. In B. S.McCrady \& E. E.Epstein (Eds.), Addictions: A comprehensive guidebook (pp. 121-140). New York: Oxford University Press.

Stephens, R. S., \& Roffman, R. A. (1993). Adult marijuana dependence. In J. S.Baer, G. A.Marlatt, \& R. J.McMahon (Eds.), Addictive behaviors across the lifespan: Prevention, treatment and policy issues (pp. 202-218). Newbury Park, CA: Sage.

Stephens, R. S., Roffman, R. A., \& Simpson, E. E. (1993). Adult marijuana users seeking treatment. Journal of Consulting and Clinical Psychology, 61, 1100-1104.

Stephens, R. S., Roffman, R. A., \& Simpson, E. E. (1994). Treating adult marijuana dependence: A test of the relapse prevention model. Journal of Consulting and Clinical Psychology, 62, 9299.

Substance Abuse and Mental Health Services Association, Office of Applied Studies. (1999). National admissions to substance abuse treatment services: The Treatment Episode Data Set (TEDS) 1992-1997. (DHHS Publication No. 99-3324). Washington, DC: U.S. Government Printing Office.

Tashkin, D. P., Fligiel, S., Wu, T., Gong, H., Jr., Barbers, R. G., Coulson, A. H., Simmons, M. S., \& Beals, T. F. (1990). Effects of habitual use of marijuana and/or cocaine on the lung. In C. N.Chiang \& R. L.Hawks (Eds.), Research findings on smoking of abused substances (National 
Institute on Drug Abuse Research Monograph No. 99; DHHS Publication No. 90-1690). Washington, DC: U. S. Government Printing Office. 